Published in Kinésithérapie, la Revue, 2018, vol. 18(194),

pp. 51-52 which should be cited to refer to this work

\title{
Un test fonctionnel: la Short Physical Performance Battery (SPPB)
}

\author{
Dominique Monnin ${ }^{a}$, Simone Gafner ${ }^{b, c}$, Lara Allet ${ }^{b, c}$ \\ a Rue des Marchands 16, CH-2900 Porrentruy, Suisse \\ ${ }^{b}$ Haute École de Santé, Filière de physiothérapie, Rue des Caroubiers 25, CH-1227 Carouge, Suisse \\ c Hôpitaux universitaires et Université de Genève, Département de Médecine communautaire, des \\ premiers recours et des urgences, Rue Gabrielle-Perret-Gentil 4, CH-1211 Genève 14, Suisse
}

Auteur correspondant : D. Monnin, Rue des Marchands 16, 2900 Porrentruy, Suisse.

Adresse e-mail : dogemo.physio@gmail.com

\section{Description}

La Short Physical Performance Battery (SPPB) a été élaborée en 1994 (1). Utilisée au départ pour mesurer les capacités fonctionnelles des personnes âgées autonomes ou hospitalisées, elle l'est aujourd'hui aussi dans de multiples domaines.

\section{Utilisation:}

Paramètres mesurés Capacités fonctionnelles des membres inférieurs: équilibration statique, vitesse de marche et relever d'une chaise

Description Le score se compose de l'addition des résultats de 3 sous-tests : capacité de garder l'équilibre pendant 10 secondes dans 3 positions (pieds joints côte à côte, en semi-tandem et en tandem), temps pour marcher 4 mètres, temps pour se relever 5 fois d'une chaise (2). Chaque tâche est évaluée entre 0 (incapacité d'effectuer la tâche) et 4 points (meilleur niveau de performance) ; le score total va donc de 0 à 12 points. Plus le score est élevé, meilleures sont les capacités fonctionnelles.

Score Les patients qui obtiennent 0 à 3 points dans le score final sont très largement handicapés dans les activités de la vie quotidienne, ils le sont modérément entre 4 et 6 points, peu entre 7 et 9 points, presque pas ou pas entre 10 et 12 points $(1,3)$.

Utilisation clinique Un score de SPPB bas prédit une diminution de la mobilité $(3,4)$, un risque de chute (5), une perte d'indépendance dans les activités quotidiennes $(3,6)$, une dépendance liée à la mobilité (3), un déclin de la santé (7), une augmentation de la durée des séjours hospitaliers (8), une ré-hospitalisation $(6,7)$, une entrée précoce en maison de retraite (1) et un décès précoce $(1$, $6,9)$.

Des études montrent que la SPPB est aussi un outil adéquat pour mesurer les capacités fonctionnelles de patients atteints de BPCO (10), d'infarctus ou insuffisance cardiaque (11), de sclérose en plaques (12) ou d'un AVC (13) ou d'une fracture du col du fémur (13).

Formation La SPPB demande peu de formation, pour les professionnels comme pour les patients.

Temps requis

Matériel 5-10 minutes

Nota bene Une chaise, un chronomètre, un couloir d'au moins 6 mètres La procédure du test n'est pas (encore) traduite en français ; elle est disponible à ce lien : http://www.mcroberts.nl/wpcontent/uploads/2016/11/SPPB form.pdf, le tableau récapitulatif à ce lien : http://www.toutsurlasarcopenie.fr/depistage/sppb/.

\section{Propriétés psychométriques}


$\mathrm{CIC}$ du test de marche : entre 0.75 et $0.90, \mathrm{CIC}$ du relever de chaise : entre 0.73 et $0.78, \mathrm{CIC}$ du test d'équilibration : entre 0.55 et 0.75 , selon l'état de santé des sujets (16). Chacun des trois tests peut être utilisé séparément (2), mais on préférera un autre test (Berg balance Scale ou Reaching test) pour évaluer spécifiquement l'équilibration.

Pour la prévention des chutes, la SPPB distingue mieux les patients chuteurs des non-chuteurs que le Timed up-and-go ou le Performance oriented mobility assessment (POMA ou test de Tinetti) (Allet L. et al., publication en cours).

Validité

La SPPB possède un niveau élevé de validité (CIC: 0.88-0.9) pour mesurer les capacités fonctionnelles d'une population âgée autonome $(11,17)$.

Différence minimale détectable (DMD):

Variation faible mais cliniquement significative : $0.5 \mathrm{pt}$; variation substantielle cliniquement significative : 1 pt (18).

Limites

La SPPB présente un effet plancher et un effet plafond; elle n'est pas indiquée pour des personnes qui possèdent un niveau de performances fonctionnelles très faible ou très élevé $(3,4)$.

\section{Commentaire}

La SPPB est un outil simple, rapide et efficace pour mesurer les capacités fonctionnelles des membres inférieurs de personnes âgées. Elle s'effectue en un temps compatible avec l'activité clinique, en pratique ambulatoire comme en institution, avec un minimum de matériel. Elle est aussi de plus en plus utilisée en recherche.

Il s'agit d'un outil qui mesure bien l'activité au sens où la décrit la Classification internationale du fonctionnement, tout à fait indiqué pour le suivi de la réadaptation.

\section{Déclaration de liens d'intérêts}

Les auteurs déclarent ne pas avoir de liens d'intérêts.

\section{Références}

1. Guralnik JM, Simonsick EM, Ferrucci L, Glynn RJ, Berkman LF, Blazer DG, et al. A short physical performance battery assessing lower extremity function: association with self-reported disability and prediction of mortality and nursing home admission. J Gerontol. 1994;49(2):M85-94. 2. Puthoff ML. Outcome measures in cardiopulmonary physical therapy: short physical performance battery. Cardiopulm Phys Ther J. 2008;19(1):17-22.

3. Guralnik JM, Ferrucci L, Simonsick EM, Salive ME, Wallace RB. Lower-extremity function in persons over the age of 70 years as a predictor of subsequent disability. N Engl J Med. 1995;332(9):556-61.

4. Vasunilashorn S, Coppin AK, Patel KV, Lauretani F, Ferrucci L, Bandinelli S, et al. Use of the Short Physical Performance Battery Score to predict loss of ability to walk 400 meters: analysis from the InCHIANTI study. J Gerontol A Biol Sci Med Sci. 2009;64(2):223-9.

5. Veronese N, Bolzetta F, Toffanello ED, Zambon S, De Rui M, Perissinotto E, et al. Association between Short Physical Performance Battery and falls in older people: the Progetto Veneto Anziani Study. Rejuvenation Res. 2014;17(3):276-84.

6. Volpato S, Cavalieri M, Sioulis F, Guerra G, Maraldi C, Zuliani G, et al. Predictive value of the Short Physical Performance Battery following hospitalization in older patients. J Gerontol A Biol Sci Med Sci. 2011;66(1):89-96.

7. Studenski S, Perera S, Wallace D, Chandler JM, Duncan PW, Rooney E, et al. Physical performance measures in the clinical setting. J Am Geriatr Soc. 2003;51(3):314-22. 
8. Fisher S, Ottenbacher KJ, Goodwin JS, Graham JE, Ostir GV. Short Physical Performance Battery in hospitalized older adults. Aging Clin Exp Res. 2009;21(6):445-52.

9. Pavasini R, Guralnik J, Brown JC, di Bari M, Cesari M, Landi F, et al. Short Physical Performance Battery and all-cause mortality: systematic review and metaanalysis. BMC Med 2016;22(14):215.

10. Bernabeu-Mora R, Medina-Mirapeix F, Llamazares-Herran E, Garcia-Guillamon G, GimenezGimenez LM, Sanchez-Nieto JM. The Short Physical Performance Battery is a discriminative tool for identifying patients with COPD at risk of disability. Int J Chron Obstruct Pulmon Dis. 2015;10:2619-26. 11. Ostir GV, Volpato S, Fried LP, Chaves P, Guralnik JM. Reliability and sensitivity to change assessed for a summary measure of lower body function: results from the Women's Health and Aging Study. J Clin Epidemiol. 2002;55(9):916-21.

12. Motl RW, Learmonth YC, Wojcicki TR, Fanning J, Hubbard EA, Kinnett-Hopkins D, et al. Preliminary validation of the short physical performance battery in older adults with multiple sclerosis: secondary data analysis. BMC Geriatr. 2015;15:157.

13. Stookey AD, Katzel LI, Steinbrenner G, Shaughnessy M, Ivey FM. The short physical performance battery as a predictor of functional capacity after stroke. J Stroke Cerebrovasc Dis. 2014;23(1):130-5.

14. Latham NK, Harris BA, Bean JF, Heeren T, Goodyear C, Zawacki S, et al. Effect of a homebased exercise program on functional recovery following rehabilitation after hip fracture: a randomized clinical trial. JAMA. 2014;311(7):700-8.

15. Gomez JF, Curcio CL, Alvarado B, Zunzunegui MV, Guralnik J. Validity and reliability of the Short Physical Performance Battery (SPPB): a pilot study on mobility in the Colombian Andes. Colomb Med (Cali). 2013;44(3):165-71.

16. Freire AN, Guerra RO, Alvarado B, Guralnik JM, Zunzunegui MV. Validity and reliability of the short physical performance battery in two diverse older adult populations in Quebec and Brazil. J Aging Health. 2012;24(5):863-78.

17. Freiberger E, de Vreede P, Schoene D, Rydwik E, Mueller V, Frandin K, et al. Performancebased physical function in older community-dwelling persons: a systematic review of instruments. Age Ageing. 2012;41(6):712-21.

18. Perera S, Mody SH, Woodman RC, Studenski SA. Meaningful change and responsiveness in common physical performance measures in older adults. J Am Geriatr Soc. 2006;54(5):743-9. 